\title{
Exponential trends in flowback chemistry from a hydraulically stimulated deep geothermal borehole in granite; Pohang, South Korea
}

\author{
David Banks ${ }^{1, *}$, Neil Burnside ${ }^{1}$, Rob Westaway $^{1}$, Günter Zimmermann ${ }^{2}$, and Hannes \\ Hofmann ${ }^{2}$ \\ ${ }^{1}$ School of Engineering, University of Glasgow, Glasgow, G12 8QQ, United Kingdom \\ ${ }^{2}$ Deutsches GeoForschungsZentrum (GFZ), Telegrafenberg, 14473 Potsdam, Germany
}

\begin{abstract}
Samples of flowback water from a $4.3 \mathrm{~km}$ deep geothermal borehole in granite (Pohang, South Korea) were collected following a period of hydraulic stimulation by injection of surface water. Electrical conductivity, temperature and water chemistry of the flowback water were measured. To a first approximation, the data conform closely to a simple 'mixing tank' model, with an exponential trend between two end members: an initial injected surface water to a more brackish 'resident groundwater' composition. Significant deviation from the 'mixing tank' trend would be an indication of significant recent water-rock interaction or other anomalous factors. Such a deviation can tentatively be seen in $\mathrm{Na}^{+} / \mathrm{Cl}^{-}$data, especially between 88 and $200 \mathrm{~m}^{3}$ flowback (2.8 to $8.8 \mathrm{hr}$ ).
\end{abstract}

\section{The Pohang site}

The Pohang geothermal site is located c. $10 \mathrm{~km}$ north of Pohang city, on the east coast of the Korean Peninsula at $129^{\circ} 22^{\prime} 46.08^{\prime \prime}$ E longitude $36^{\circ} 06^{\prime} 23.34^{\prime \prime} \mathrm{N}$ latitude. The geothermal project comprises two boreholes (PX-1 and PX-2) drilled through c. $2.3 \mathrm{~km}$ of Quaternary, Tertiary and Cretaceous sediments and sedimentary / volcanic rocks, into a Permian granodiorite containing mafic dykes $[1,2]$. The site was originally intended as an Enhanced Geothermal System (EGS) installation, where hydraulic stimulation has attempted to create a fracture connection in the granite between the two boreholes, forming a heat exchange surface [3].

Well PX-1 has been completed to a total length of $4348 \mathrm{~m}$ and a vertical depth (TVD) of $4127 \mathrm{~m}$, and deviates c. $600 \mathrm{~m}$ to the WNW. The total volume of PX-1 borehole is calculated as $85 \mathrm{~m}^{3}$, with $74 \mathrm{~m}^{3}$ in the cased portion and $11 \mathrm{~m}^{3}$ in the lowest (open hole) section. Stimulation took place in December 2016 and in August 2017 in well in well PX-1, and in February 2016 and April and September 2017 in well PX-2. A subsequent magnitude $\left(\mathrm{M}_{\mathrm{W}}\right) 5.5$ earthquake in November 2017 [4], with hypocentre no more than hundreds of metres from the injection points, has halted further operations, while possible causes are

* Corresponding author: david.banks@ glasgow.ac.uk 
investigated [4]. The focus of this paper is the flowback water produced following the stimulation of well PX-1 in August 2017.

Between $7^{\text {th }}$ and $14^{\text {th }}$ August $2017,1756 \mathrm{~m}^{3}$ of local surface water, sourced from a pond, were injected into PX-1. Flowback commenced at 09:34 on $14^{\text {th }}$ August at a rate in excess of $6 \mathrm{~L} / \mathrm{s}$ (Fig. 1). By $20^{\text {th }}$ August, $1028 \mathrm{~m}^{3}$ had been recovered, and the flowback rate had decreased to c. $0.5 \mathrm{~L} / \mathrm{s}$. Following this flowback period, well PX-1 was subject to a production test to ascertain any transmissivity increase. In total the volume of flowback water recovered was $1773 \mathrm{~m}^{3}$.

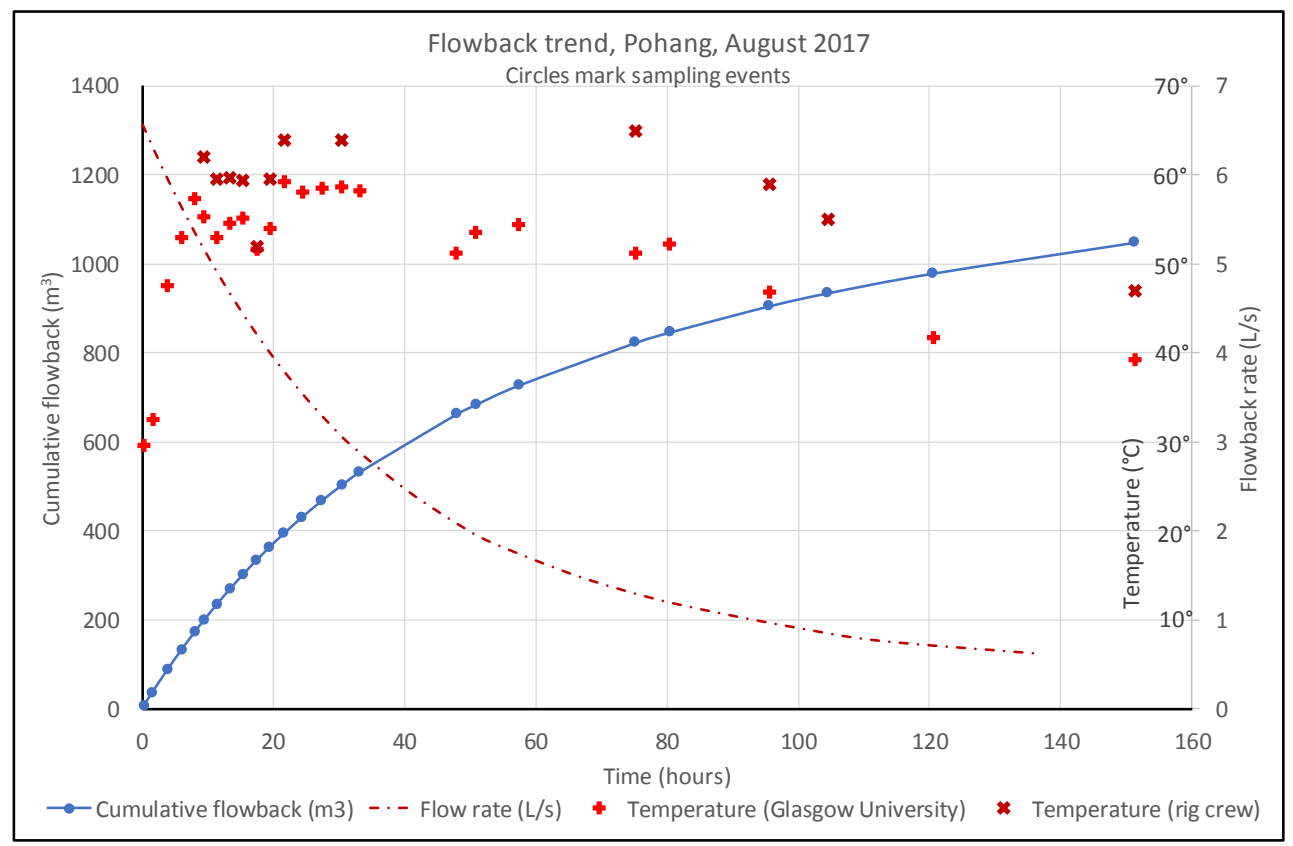

Fig. 1. Cumulative flowback and estimated flowback rate from PX-1, with time (from 0934 on $14^{\text {th }}$ August 2017). Circles show sampling times. Crosses show temperature, which decreases as flowback rate slows (presumably due to greater conductive heat losses during uphole flow).

\section{Methods}

During flowback, water samples were collected; temperature and electrical conductivity (EC - Fig. 2) were measured using an Ultrameter II 6PFC instrument (Myron L Company, Carlsbad, California, with results corrected to a standard temperature of $25^{\circ} \mathrm{C}$ ), initially at the point where the pipe leading from the well head discharged into the mud tank, later (after flow rates had decreased) at the well head. Temperature measurements were also made by members of the rig crew, who reported typical water temperatures of 50 to $65^{\circ} \mathrm{C}$.

Subsequently at University of Glasgow water samples were analysed for a variety of parameters. Those of direct relevance here are $\mathrm{Na}^{+}$and $\mathrm{Cl}^{-}$, which were analysed by ion chromatography. An aliquot of sample was run for anions on an ICS-900 system. A second aliquot was filtered using a $0.45 \mu \mathrm{m}$ filter capsule, acidified with concentrated Ultrapur $\mathrm{HNO}_{3}$ and then run for cations on an ICS-1100 ion chromatograph. 


\section{Results}

The surface water used for injection can be characterised as a $\mathrm{Ca}-(\mathrm{Na})-\mathrm{SO}_{4}-(\mathrm{Cl})$ water of circumneutral $\mathrm{pH}$. EC $\left(25^{\circ} \mathrm{C}\right)$ varied from 897 to $1322 \mu \mathrm{S} / \mathrm{cm}$, while $\mathrm{Na}^{+}$and $\mathrm{Cl}^{-}$varied from 51 to $120 \mathrm{mg} / \mathrm{L}$ and 56 to $163 \mathrm{mg} / \mathrm{L}$, respectively (5 samples).

The initial flowback water was, unsurprisingly, similar to the injection water and one can surmise that the first two points on Figures 2 and 3 (i.e. $<85 \mathrm{~m}^{3}$ cumulative flowback $=$ borehole volume) comprised water that had never left the borehole and so did not enter the granite. Subsequently, the salinity of the water increased relatively sharply.

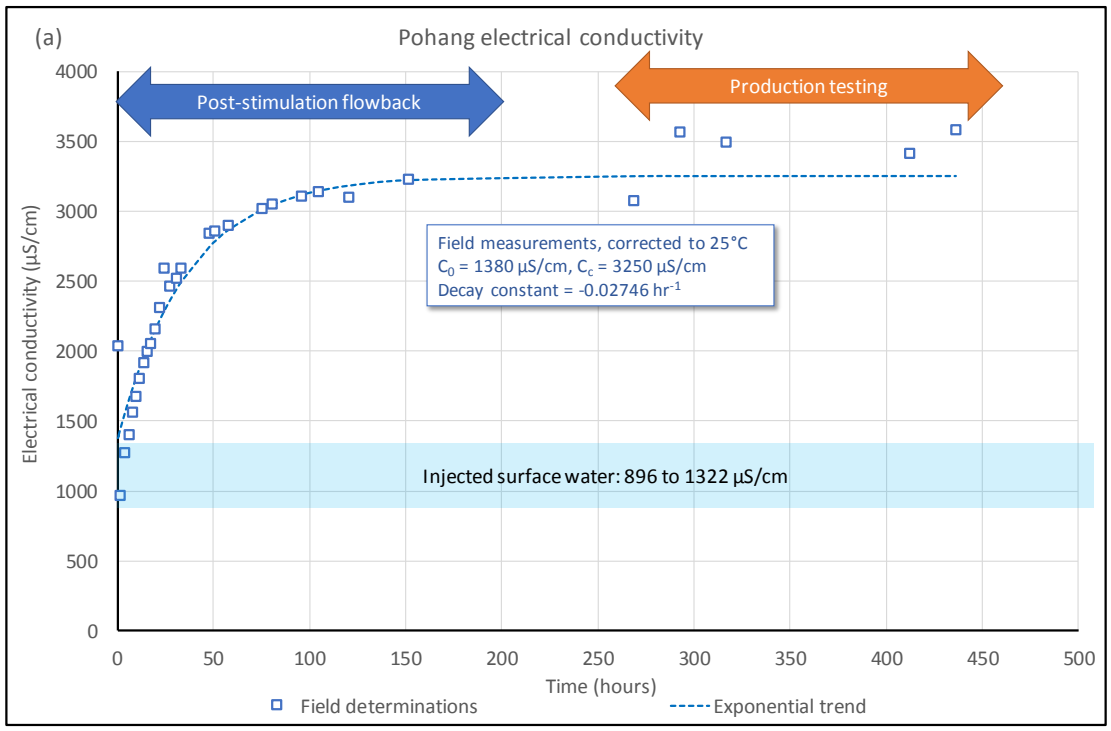

Fig. 2. Electrical conductivity (EC, corrected to $25^{\circ} \mathrm{C}$ ), sodium and chloride concentrations in flowback water from PX-1 versus time. Dashed lines show best fit exponential curves, ignoring first data point and production test data.

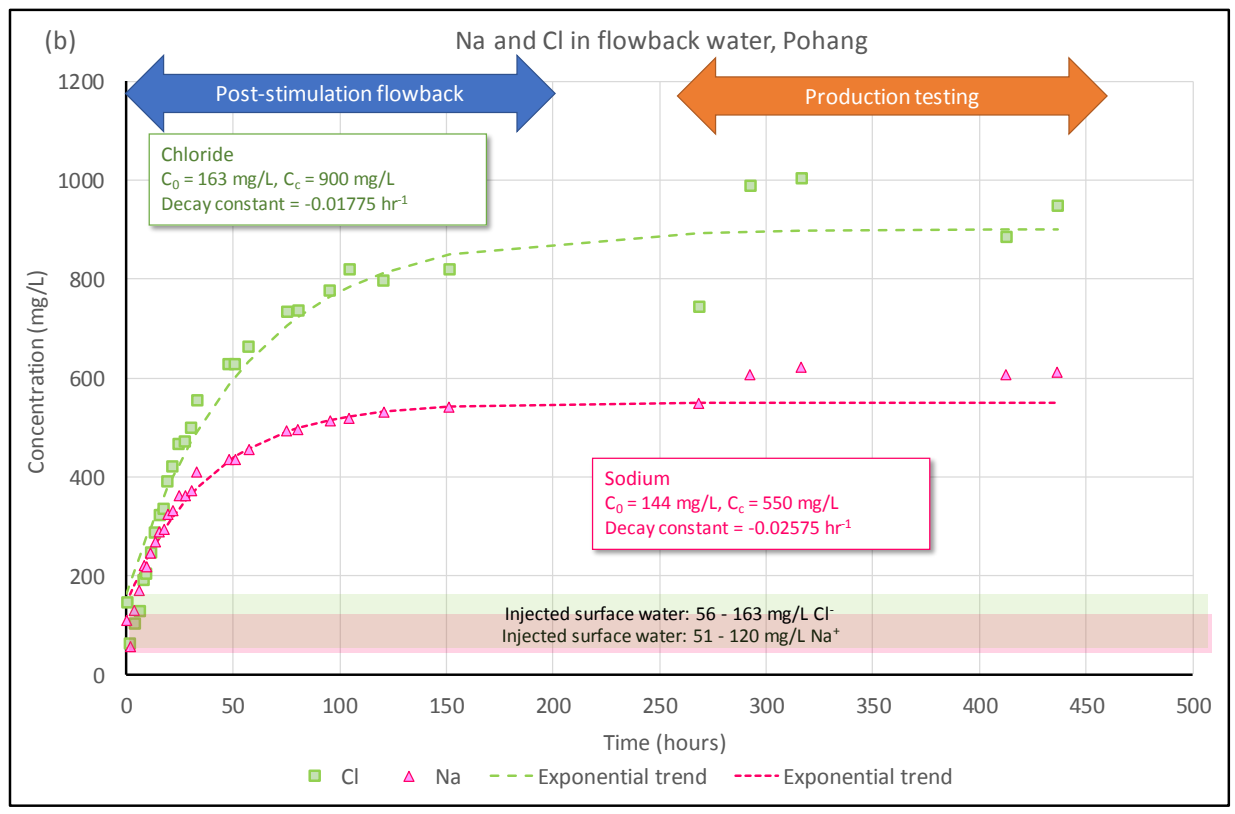



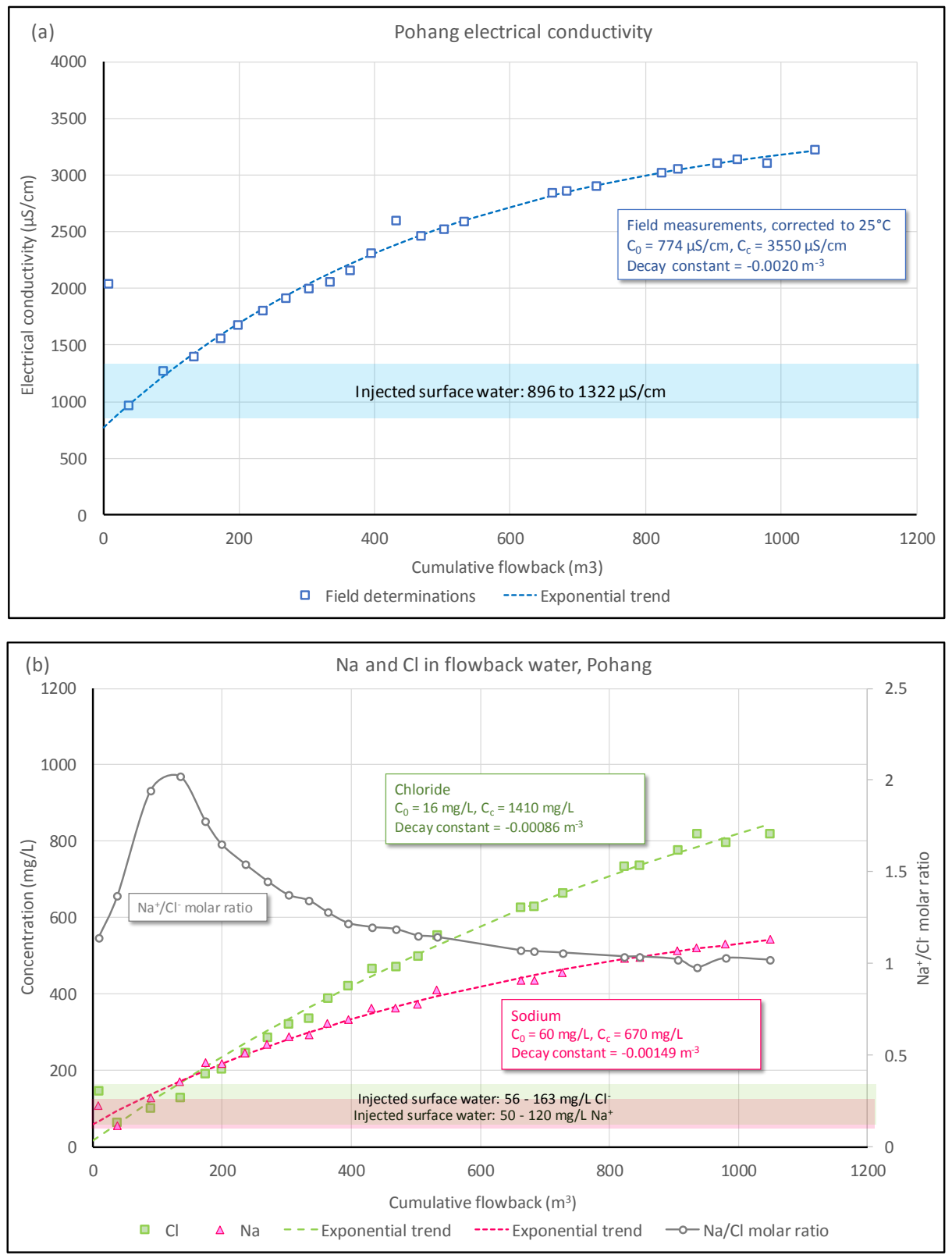

Fig. 3. Electrical conductivity (EC), sodium and chloride concentrations and $\mathrm{Na}^{+} / \mathrm{Cl}^{-}$molar ratio in flowback water from PX-1 versus cumulative flowback. Dashed lines $=$ best fit exponential curves.

The gradient of the increase rapidly shallowed out, with the appearance of approaching a "ceiling" with increasing time. Therefore, one can assume that the initial concentration $C_{0}$ represents the injected water, and the "ceiling" concentration $C_{c}$ represents groundwater entering from the granite. It is worth stating that the quality of the water already residing in the granite (i.e. $C_{c}$ ) may in principle have been affected by previous cycles of injection and hydraulic stimulation. $C_{c}$ cannot therefore be assumed to be the "natural" in situ granite composition. 


\section{Trends}

The trends (Figs. 2 and 3) bear a strong resemblance to a 'mixing tank' model between two end members, where an initial concentration tends towards a final concentration in an exponential manner [5]. Best fit exponential trends of the form:

$$
C=C_{c}-\left(C_{c^{-}} C_{0}\right) \times \mathrm{e}^{m x}
$$

have been fitted to the flowback data, where $x$ is a parameter used to quantify the flowback (time in Fig. 2 and volume in Fig. 3), $C=$ concentration or conductivity, $C_{c}$ and $C_{0}$ are the ceiling and initial values of $C$, and $m$ is a decay constant $\left(\mathrm{hr}^{-1} \mathrm{or} \mathrm{m}^{-3}\right.$ depending on $x$ ).

One can observe that the modelled fit is relatively good, although the later data from production testing do not fit the trend especially well, especially for $\mathrm{Na}^{+}$(Fig. 2). Given that the flow rate changes with time, it is regarded as more meaningful to plot the parameters versus cumulative flowback (Fig. 3). In this case, the plots are much more 'linear' in nature, although still with a gradient that declines with time. In this case, it is far less clear that an exponential model is the best model to apply, but exponential trend curves can still be fitted to the data. These, however, produce much higher 'ceilings' $C_{c}$ and the confidence in this result is lower. The decay constants are rather divergent (which they should not be if the 'mixing tank' analogy were entirely valid). Decay constants vary from $-0.0020 \mathrm{~m}^{-3}$ for EC to $-0.0015 \mathrm{~m}^{-3}$ for $\mathrm{Na}^{+}$and $-0.0009 \mathrm{~m}^{-3}$ for $\mathrm{Cl}^{-}$.

\section{Conclusion}

Conservative parameters in flowback water appear to approximately adhere to an exponential mixing model between two end members (the injection water and a resident groundwater). Such trends for conservative parameters such as $\mathrm{Cl}^{-}$and EC can be very useful in establishing a baseline against which other parameters can be compared, to evaluate the extent of any water-rock interaction on the injected fluid. Close inspection of Figure 3 reveals, for example, that in the early phases of flowback (particularly at cumulative volume $=88$ to $200 \mathrm{~m}^{3}$ ), sodium actually exceeds chloride in concentration, before returning to a much lower (seawater-like) ratio. This may, in turn, suggest some degree of acquisition of sodium by the injected fluid reacting with silicate minerals, or it may simply reflect variations in quality of the injected fluid over time. Dissolved silica concentrations (not presented here) also show a much more rapid rise with cumulative flowback than a pure mixing model would suggest, indicating silicate weathering or silica dissolution by the injected water.

Acknowledgements. This work was funded by the EU H2020 LCE 'DESTRESS' project (EC691728). We thank Anne McGarrity for analyses and DESTRESS co-workers for helpful discussions. We especially thank Paul Younger, who led UoG involvement in DESTRESS and helped to secure the funding for this work.

\section{References}

1. T. Lee, et al., Proc. World Geotherm Cong, Melbourne, Paper 31025 (2015)

2. K.-S. Yoon, et al., Proc. World Geotherm Cong, Melbourne, Paper 06034 (2015)

3. S. Park, et al., Proc. Eng 191, 829-837 (2017)

4. F. Grigoli, et al., Science 360, 1003-1006 (2018)

5. G. Gzyl, D. Banks, J. Contam Hydrol. 92, 66-86 (2007) 